\title{
A quantitative approach to perfect one-factorizations of complete bipartite graphs*
}

\author{
Natacha Astromujoff \\ Departamento de Matemáticas \\ Universidad de Chile \\ Santiago, Chile
}

\author{
Martín Matamala \\ Department of Mathematical Engineering and \\ Centre for Mathematical Modeling (UNMI 2807 CNRS) \\ Universidad de Chile \\ Santiago, Chile
}

Submitted: Feb 18, 2014; Accepted: Mar 11, 2015; Published: Mar 23, 2015

Mathematics Subject Classification: 05B15, 05C70

\begin{abstract}
Given a one-factorization $\mathcal{F}$ of the complete bipartite graph $K_{n, n}$, let $\operatorname{pf}(\mathcal{F})$ denote the number of Hamiltonian cycles obtained by taking pairwise unions of perfect matchings in $\mathcal{F}$. Let $\operatorname{pf}(n)$ be the maximum of $\operatorname{pf}(\mathcal{F})$ over all one-factorizations $\mathcal{F}$ of $K_{n, n}$. In this work we prove that $\operatorname{pf}(n) \geqslant n^{2} / 4$, for all $n \geqslant 2$.
\end{abstract}

\section{Perfect one-factorizations}

A one-factorization of a graph $G$ is a partition of its set of edges into perfect matchings (see Wallis [8]). The union of two distinct perfect matchings (or 1-factors) $A$ and $B$ in any one-factorization of a graph $G$ induces a spanning subgraph $G_{A, B}$ which is the vertex disjoint union of even length cycles. When this graph $G_{A, B}$ is a Hamiltonian cycle, the pair $A, B$ is called a perfect pair. A one-factorization $\mathcal{F}$ is perfect if the graph induced by every two distinct perfect matchings in $\mathcal{F}$ is a Hamiltonian cycle (see Seah [7]).

The perfect one-factorization conjecture states that for any positive integer $n$, the complete graph $K_{2 n}$ admits a perfect one-factorization. The conjecture seems to be first explicitly mentioned in [3] although it was mentioned informally in [5]. Additionally, in [6] determining the set of all $n$ for which $K_{2 n}$ has a perfect one-factorization appears as a problem. There are only two infinite families for which it is known that $K_{2 n}$ has a perfect one-factorization: $n \in\{(p+1) / 2, p\}$, for any odd prime $p[1,5]$.

A quantitative version of this conjecture was pursued by Wagner [9]. For even $n$, Wagner defined $c(n)$ as the maximum over all one-factorizations $\mathcal{F}$ of $K_{n}$ of the number of perfect pairs in $\mathcal{F}$ and for odd $n$, he defined $c(n)=c(n+1)$. Hence, for any even

\footnotetext{
*This work was partially supported by program Basal-CMM (M.M.) and Núcleo Milenio Información y Coordinación en Redes ICM/FIC RC130003 (N.A. and M.M.).
} 
$n$ such that $K_{n}$ has a perfect one-factorization, $c(n)=\left(\begin{array}{c}n-1 \\ 2\end{array}\right)$. Wagner showed that $c(n m) \geqslant 2 c(n) c(m)$ whenever $m$ and $n$ are odd and coprime and $c(n) \geqslant n \phi(n) / 2$, where $n$ is odd and $\phi$ is the Euler totient function.

In this work we undertake a similar analysis for one-factorizations of complete bipartite graphs. For a positive integer $n$ we define $\operatorname{pf}(n)$ to be the maximum over all one-factorizations $\mathcal{F}$ of $K_{n, n}$ of the number of perfect pairs in $\mathcal{F}$. As before, if the complete bipartite graph $K_{n, n}$ has a perfect one-factorization, then $\operatorname{pf}(n)=\left(\begin{array}{l}n \\ 2\end{array}\right)$. It is known that if $K_{n}$ has a perfect one factorization then $K_{n-1, n-1}$ has a perfect one-factorization as well (see Wanless and Ihrig [12]). Then, from what we know for complete graphs, two infinite families of complete bipartite graphs are known to admit a perfect one-factorization: $K_{p, p}$ and $K_{2 p-1,2 p-1}$, for each odd prime $p$. In [2] it was proved that for each odd prime $p$, the complete bipartite graph $K_{p^{2}, p^{2}}$ also admits a perfect one-factorization. This evidence leads to the following version of the perfect one-factorization conjecture for complete bipartite graphs, first stated by Wanless in [10].

Conjecture 1 For every odd $n \geqslant 3, \operatorname{pf}(n)=\left(\begin{array}{l}n \\ 2\end{array}\right)$.

In contrast, for each even $n$ it is known that $\operatorname{pf}(n) \leqslant n^{2} / 4$ and that this upper bound is achieved for each $n=2 p$, where $p$ is an odd prime [11].

Our results are presented in the language of Latin rectangles since it makes the presentation easier. Given two positive integers $m$ and $n$ with $m \leqslant n$, a Latin rectangle $L$ of size $m \times n$ is a matrix with $m$ rows and $n$ columns filled with symbols from an alphabet $\Sigma_{L}$ of size $n$, such that each row contains each symbol in $\Sigma_{L}$ once, and each column contains each symbol in $\Sigma_{L}$ at most once. When $m=n$ a Latin rectangle is called a Latin square of order $n$. Two rows $i$ and $j$ of a Latin rectangle $L$ of size $m \times n$ form a perfect pair if $i \neq j$ and the permutation $L_{i, j}$, which assigns to the symbol $x$ in row $i$ and column $k$ the symbol $y$ in row $j$ and column $k$, is a cyclic permutation. We denote by $\operatorname{pf}(R)$ the number of perfect pairs in a Latin rectangle $R$ and by $\operatorname{pf}(m, n)$ the maximum of $\operatorname{pf}(R)$ over all Latin rectangles $R$ of size $m \times n$. Then $\operatorname{pf}(m, n) \leqslant\left(\begin{array}{c}m \\ 2\end{array}\right)$. When a Latin rectangle of size $m \times n$ achieves this upper bound, it is called a perfect or pan-Hamiltonian Latin rectangle $([4],[10])$. It is known that there is a one-to-one correspondence between perfect Latin squares of order $n$ and perfect one-factorizations of $K_{n, n}$ ([12]). From this relation it is easy to derive that $\operatorname{pf}(n, n)$ is in fact the same as $\operatorname{pf}(n)$.

Our main result is that $\operatorname{pf}(n) \geqslant n^{2} / 4$ for each $n \geqslant 2$. The proof is split into the cases $n$ even and $n$ odd. In Section 2 we explicitly construct, for each even $n$, a Latin square $D$ of order $n$ such that $\operatorname{pf}(D)=n^{2} / 4$. In Section 3 we prove that for each odd $n, \operatorname{pf}(n)>n^{2} / 4$.

\section{Even order case}

As we previously mentioned, in [11] it was proved that for each even $n, \operatorname{pf}(n) \leqslant n^{2} / 4$. In the next result we prove that equality holds.

Theorem 1 For each even $n, \operatorname{pf}(n)=n^{2} / 4$. 
Proof: We only need to prove that for each even $n$, there is a Latin square of order $n$ having $n^{2} / 4$ perfect pairs.

In the rest of this proof all calculations are carried out in $\mathbb{Z}_{n / 2}$. Let $A, B$ and $C$ be the following Latin squares of orders $n / 2$ with $\Sigma_{A}=\Sigma_{B}=\Sigma_{C}=\mathbb{Z}_{n / 2}$. For each $i, j \in \mathbb{Z}_{n / 2}$,

- $A(i, j)=j+i \bmod n / 2$.

- $B(i, j)=j+i+1 \bmod n / 2$.

- $C(i, j)=j-i \bmod n / 2$.

Let $D$ be the Latin square of order $n$ with $\Sigma_{D}=\mathbb{Z}_{n / 2} \times\{0,1\}$ and defined as follows. For $a, c \in \mathbb{Z}_{n / 2}$ and $b, d \in\{0,1\}$,

- $D((a, b),(c, d))=(C(a, c), 0)$ when $b=d=0$,

- $D((a, b),(c, d))=(C(a, c), 1)$ when $b=1$ and $d=0$,

- $D((a, b),(c, d))=(A(a, c), 1)$ when $b=0$ and $d=1$, and

- $D((a, b),(c, d))=(B(a, c), 0)$ when $b=d=1$.

In the definition of $D$, rows and columns are indexed by symbols in $\Sigma_{D}$ (see Figure 1 ).

\begin{tabular}{|llllllllll|llllllllll|}
\hline 00 & 10 & 20 & 30 & 40 & 50 & 60 & 70 & 80 & 90 & 01 & 11 & 21 & 31 & 41 & 51 & 61 & 71 & 81 & 91 \\
90 & 00 & 10 & 20 & 30 & 40 & 50 & 60 & 70 & 80 & 11 & 21 & 31 & 41 & 51 & 61 & 71 & 81 & 91 & 01 \\
80 & 90 & 00 & 10 & 20 & 30 & 40 & 50 & 60 & 70 & 21 & 31 & 41 & 51 & 61 & 71 & 81 & 91 & 01 & 11 \\
70 & 80 & 90 & 00 & 10 & 20 & 30 & 40 & 50 & 60 & 31 & 41 & 51 & 61 & 71 & 81 & 91 & 01 & 11 & 21 \\
60 & 70 & 80 & 90 & 00 & 10 & 20 & 30 & 40 & 50 & 41 & 51 & 61 & 71 & 81 & 91 & 01 & 11 & 21 & 31 \\
50 & 60 & 70 & 80 & 90 & 00 & 10 & 20 & 30 & 40 & 51 & 61 & 71 & 81 & 91 & 01 & 11 & 21 & 31 & 41 \\
40 & 50 & 60 & 70 & 80 & 90 & 00 & 10 & 20 & 30 & 61 & 71 & 81 & 91 & 01 & 11 & 21 & 31 & 41 & 51 \\
30 & 40 & 50 & 60 & 70 & 80 & 90 & 00 & 10 & 20 & 71 & 81 & 91 & 01 & 11 & 21 & 31 & 41 & 51 & 61 \\
20 & 30 & 40 & 50 & 60 & 70 & 80 & 90 & 00 & 10 & 81 & 91 & 01 & 11 & 21 & 31 & 41 & 51 & 61 & 71 \\
10 & 20 & 30 & 40 & 50 & 60 & 70 & 80 & 90 & 00 & 91 & 01 & 11 & 21 & 31 & 41 & 51 & 61 & 71 & 81 \\
\hline 01 & 11 & 21 & 31 & 41 & 51 & 61 & 71 & 81 & 91 & 10 & 20 & 30 & 40 & 50 & 60 & 70 & 80 & 90 & 00 \\
91 & 01 & 11 & 21 & 31 & 41 & 51 & 61 & 71 & 81 & 20 & 30 & 40 & 50 & 60 & 70 & 80 & 90 & 00 & 10 \\
81 & 91 & 01 & 11 & 21 & 31 & 41 & 51 & 61 & 71 & 30 & 40 & 50 & 60 & 70 & 80 & 90 & 00 & 10 & 20 \\
71 & 81 & 91 & 01 & 11 & 21 & 31 & 41 & 51 & 61 & 40 & 50 & 60 & 70 & 80 & 90 & 00 & 10 & 20 & 30 \\
61 & 71 & 81 & 91 & 01 & 11 & 21 & 31 & 41 & 51 & 50 & 60 & 70 & 80 & 90 & 00 & 10 & 20 & 30 & 40 \\
51 & 61 & 71 & 81 & 91 & 01 & 11 & 21 & 31 & 41 & 60 & 70 & 80 & 90 & 00 & 10 & 20 & 30 & 40 & 50 \\
41 & 51 & 61 & 71 & 81 & 91 & 01 & 11 & 21 & 31 & 70 & 80 & 90 & 00 & 10 & 20 & 30 & 40 & 50 & 60 \\
31 & 41 & 51 & 61 & 71 & 81 & 91 & 01 & 11 & 21 & 80 & 90 & 00 & 10 & 20 & 30 & 40 & 50 & 60 & 70 \\
21 & 31 & 41 & 51 & 61 & 71 & 81 & 91 & 01 & 11 & 90 & 00 & 10 & 20 & 30 & 40 & 50 & 60 & 70 & 80 \\
11 & 21 & 31 & 41 & 51 & 61 & 71 & 81 & 91 & 01 & 00 & 10 & 20 & 30 & 40 & 50 & 60 & 70 & 80 & 90 \\
\hline
\end{tabular}

Figure 1: Latin square of order 20 achieving $\operatorname{pf}(n)=n^{2} / 4$. The symbol $(a, b)$ is represented as $a b$. 
It is easy to see that for each pair $(i, 0)$ and $(l, 1)$ in $\Sigma_{D}$ and for each $a \in \mathbb{Z}_{n / 2}$, the permutation $D_{(i, 0),(l, 1)}$ maps $(a, 0)$ to $(a-l+i \bmod n / 2,1)$ and $(a, 1)$ to $(a+l-i+$ $1 \bmod n / 2,0)$. Hence, $D_{(i, 0),(l, 1)}^{2}((a, 0))=(a+1 \bmod n / 2,0)$, for each $i, l, a \in \mathbb{Z}_{n / 2}$. Therefore, the permutation $D_{(i, 0),(l, 1)}$ is a cyclic permutation for each pair $i, l \in \mathbb{Z}_{n / 2}$. This finishes the proof.

\section{Odd order case}

From now on, for every Latin rectangle $L$ we shall assume that each column is labelled by the corresponding symbol from its first row and that each row is labelled by the corresponding symbol from its first column. Thus the first row and the first column have the same label and we shall denote this label by $\bar{L}$.

We also denote by $L_{a}$ the permutation $L_{a, \bar{L}}$, for each row $a$ of $L$. With this notation, for every two rows $a$ and $a^{\prime}$ of $L$ we have the following relations: $L_{a}^{-1}=L_{\bar{L}, a}$ and $L_{a, a^{\prime}}=$ $L_{a^{\prime}}^{-1} \circ L_{a}$.

In order to prove that $\operatorname{pf}(n) \geqslant n^{2} / 4$ for each odd $n$, we prove something slightly more general. Namely, we prove that for each pair of odd integers $m$ and $n$ with $3 \leqslant m \leqslant n$ we have that $\operatorname{pf}(m, n) \geqslant m^{2} / 4$.

When $n$ is an odd prime the result is direct since we know that there is a Latin square $N$ of order $n$ such that $\operatorname{pf}(N)=\left(\begin{array}{l}n \\ 2\end{array}\right)$. Hence, for each $m \leqslant n$ the Latin rectangle $N^{\prime}$ obtained by choosing $m$ rows of $N$ satisfies $\operatorname{pf}\left(N^{\prime}\right)=\left(\begin{array}{c}m \\ 2\end{array}\right)$.

When $n$ is an odd composite number we proceed in three steps. We first prove that $\operatorname{pf}(n) \geqslant \operatorname{pf}(p, n / p)(n / p)^{2}$, where $p$ is the smallest prime divisor of $n$. Later, we prove that if $\operatorname{pf}(n) \geqslant n^{2} / 4$, then $\operatorname{pf}(m, n) \geqslant m^{2} / 4$, for each $m \leqslant n$. Finally, by using the two first steps we prove that $\operatorname{pf}(n) \geqslant n^{2} / 4$, by induction on $n$.

\section{Construction}

Let $n$ be an odd composite number. Given any Latin rectangle $K$ of size $p \times(n / p)$, where $p$ is the smallest prime divisor of $n$, we construct a Latin square $\mathrm{T}$ of order $n$ such that $\operatorname{pf}(\mathrm{T}) \geqslant \operatorname{pf}(K)(n / p)^{2}$. We first describe the construction of $\mathrm{T}$ in terms of $K$ and give an example for $n=15$. Later, in Theorem 2, we prove that $\operatorname{pf}(\mathrm{T}) \geqslant \operatorname{pf}(K)(n / p)^{2}$.

\section{Description}

Let $L$ be the Latin square of order $n / p$ with $\Sigma_{L}=\mathbb{Z}_{n / p}$ and $\bar{L}=0 \in \mathbb{Z}_{n / p}$ given by $L(x, y)=x+y \bmod (n / p)$, where $x, y \in \mathbb{Z}_{n / p}$. Then, $L_{a, a^{\prime}}(x)=x+\left(a^{\prime}-a\right) \bmod (n / p)$ and $L_{a, a^{\prime}}^{t}(x)=x+t\left(a^{\prime}-a\right) \bmod (n / p)$, for each $x, a, a^{\prime} \in \mathbb{Z}_{n / p}$ and each $t \in \mathbb{Z}$.

Let $P$ be a Latin square of order $p$ such that $\operatorname{pf}(P)=\left(\begin{array}{l}p \\ 2\end{array}\right)$. Without loss of generality, we assume that $\Sigma_{L}=\Sigma_{K}$ and that $\Sigma_{P} \subseteq \Sigma_{L}$. This is possible because $p \leqslant n / p$.

Let $\Sigma_{\mathrm{T}}:=\Sigma_{L} \times \Sigma_{P}$ and let $\mathrm{T}^{\prime}$ be the Cartesian product of $L$ and $P$ given, for each $(a, b),(c, d) \in \Sigma_{T}$, by

$$
\mathbf{T}^{\prime}((a, b),(c, d))=(L(a, c), P(b, d)) .
$$




\begin{tabular}{|lll|lll|lll|lll|lll|l|}
\hline $\mathbf{0 0}$ & 01 & 02 & $\mathbf{1 0}$ & 11 & 12 & $\mathbf{2 0}$ & 21 & 22 & $\mathbf{3 0}$ & 31 & 32 & $\mathbf{4 0}$ & 41 & 42 \\
01 & 02 & $\mathbf{0 0}$ & 11 & 12 & $\mathbf{1 0}$ & 21 & 22 & $\mathbf{2 0}$ & 31 & 32 & $\mathbf{3 0}$ & 41 & 42 & $\mathbf{4 0}$ \\
02 & $\mathbf{0 0}$ & 01 & 12 & $\mathbf{1 0}$ & 11 & 22 & $\mathbf{2 0}$ & 21 & 32 & $\mathbf{3 0}$ & 31 & 42 & $\mathbf{4 0}$ & 41 \\
\hline $\mathbf{1 0}$ & 11 & 12 & $\mathbf{2 0}$ & 21 & 22 & $\mathbf{3 0}$ & 31 & 32 & $\mathbf{4 0}$ & 41 & 42 & $\mathbf{0 0}$ & 01 & 02 \\
11 & 12 & $\mathbf{1 0}$ & 21 & 22 & $\mathbf{2 0}$ & 31 & 32 & $\mathbf{3 0}$ & 41 & 42 & $\mathbf{4 0}$ & 01 & 02 & $\mathbf{0 0}$ \\
12 & $\mathbf{1 0}$ & 11 & 22 & $\mathbf{2 0}$ & 21 & 32 & $\mathbf{3 0}$ & 31 & 42 & $\mathbf{4 0}$ & 41 & 02 & $\mathbf{0 0}$ & 01 \\
\hline $\mathbf{2 0}$ & 21 & 22 & $\mathbf{3 0}$ & 31 & 32 & $\mathbf{4 0}$ & 41 & 42 & $\mathbf{0 0}$ & 01 & 02 & $\mathbf{1 0}$ & 11 & 12 \\
21 & 22 & $\mathbf{2 0}$ & 31 & 32 & $\mathbf{3 0}$ & 41 & 42 & $\mathbf{4 0}$ & 01 & 02 & $\mathbf{0 0}$ & 11 & 12 & $\mathbf{1 0}$ \\
22 & $\mathbf{2 0}$ & 21 & 32 & $\mathbf{3 0}$ & 31 & 42 & $\mathbf{4 0}$ & 41 & 02 & $\mathbf{0 0}$ & 01 & 12 & $\mathbf{1 0}$ & 11 \\
\hline $\mathbf{3 0}$ & 31 & 32 & $\mathbf{4 0}$ & 41 & 42 & $\mathbf{0 0}$ & 01 & 02 & $\mathbf{1 0}$ & 11 & 12 & $\mathbf{2 0}$ & 21 & 22 \\
31 & 32 & $\mathbf{3 0}$ & 41 & 42 & $\mathbf{4 0}$ & 01 & 02 & $\mathbf{0 0}$ & 11 & 12 & $\mathbf{1 0}$ & 21 & 22 & $\mathbf{2 0}$ \\
32 & $\mathbf{3 0}$ & 31 & 42 & $\mathbf{4 0}$ & 41 & 02 & $\mathbf{0 0}$ & 01 & 12 & $\mathbf{1 0}$ & 11 & 22 & $\mathbf{2 0}$ & 21 \\
\hline $\mathbf{4 0}$ & 41 & 42 & $\mathbf{0 0}$ & 01 & 02 & $\mathbf{1 0}$ & 11 & 12 & $\mathbf{2 0}$ & 21 & 22 & $\mathbf{3 0}$ & 31 & 32 \\
41 & 42 & $\mathbf{4 0}$ & 01 & 02 & $\mathbf{0 0}$ & 11 & 12 & $\mathbf{1 0}$ & 21 & 22 & $\mathbf{2 0}$ & 31 & 32 & $\mathbf{3 0}$ \\
42 & $\mathbf{4 0}$ & 41 & 02 & $\mathbf{0 0}$ & 01 & 12 & $\mathbf{1 0}$ & 11 & 22 & $\mathbf{2 0}$ & 21 & 32 & $\mathbf{3 0}$ & 31 \\
\hline
\end{tabular}

Figure 2: The symbol $(a, b)$ is represented as $a b$. In bold we emphasize those symbols in $\Sigma_{T^{\prime}}$ whose second component is $\bar{P}=0$.

For each $a \in \Sigma_{L}$ and each $b \in \Sigma_{P}$, we define the permutation $\pi_{a, b}=K_{b}^{-1} \circ L_{a}^{p}$.

Finally, we define $\mathrm{T}$ to be the $n \times n$ matrix obtained from $\mathrm{T}^{\prime}$ by changing in each row $(a, b)$ of $\mathrm{T}^{\prime}$ the symbol $(x, \bar{P})$ to $\left(\pi_{a, b}(x), \bar{P}\right)$, for each $x \in \Sigma_{L}$.

Example 1 Let $K$ be the Latin rectangle of size $3 \times 5$ with $\Sigma_{K}=\mathbb{Z}_{5}$ and $\bar{K}=0 \in \mathbb{Z}_{5}$, defined by $K(x, y)=x+y \bmod 5$, for $x \in\{0,1,2\}$ and $y \in \mathbb{Z}_{5}$. For each $b \in\{0,1,2\}$ and each $y \in \mathbb{Z}_{5}$, we have that

$$
K_{b}^{-1}(y)=K_{0, b}(y)=K(b, y)=b+y \bmod 5 .
$$

In Figure 2 we present the construction of the Latin square $\mathrm{T}^{\prime}$ of order 15 based on the two Latin squares $P$ and $L$ of sizes $p=3$ and $n / p=5$, respectively, where $\Sigma_{L}=\mathbb{Z}_{5}$ and $L(x, y)=x+y \bmod 5$, for each $x, y \in \mathbb{Z}_{5}$, and $\Sigma_{P}=\mathbb{Z}_{3}$ and $P(x, y)=x+y \bmod 3$. Additionally, $\bar{P}=0 \in \mathbb{Z}_{3}$ and $\bar{L}=0 \in \mathbb{Z}_{5}$.

We now explicitly determine the permutation $\pi_{a, b}$ for each $a \in \mathbb{Z}_{5}$ and each $b \in \mathbb{Z}_{3}$. By definition, $\pi_{a, b}$ is given by $\pi_{a, b}(x)=K_{b}^{-1} \circ L_{a}^{3}(x)$, for each $x \in \mathbb{Z}_{5}$. We know that $K_{b}^{-1}(y)=y+b \bmod 5$ and that $L_{a}^{3}(x)=x-3 a \bmod 5$. Hence $\pi_{a, b}(x)=x-3 a+b$ $\bmod 5$, for each $x \in \mathbb{Z}_{5}$.

When we apply the modifications induced by the permutations $\pi_{a, b}$ to $\mathrm{T}^{\prime}$ we obtain the Latin square $\mathrm{T}$ given in Figure 3.

Roughly speaking the role of the modifications defined by the permutations $\pi_{a, b}$ is to glue cycles of some row permutations of $\mathrm{T}^{\prime}$ into cycles of length $n$. For instance in 


\begin{tabular}{|lll|lll|lll|lll|lll|}
\hline 00 & 01 & 02 & 10 & 11 & 12 & 20 & 21 & 22 & 30 & 31 & 32 & 40 & 41 & 42 \\
01 & 02 & $\mathbf{1 0}$ & 11 & 12 & $\mathbf{2 0}$ & 21 & 22 & $\mathbf{3 0}$ & 31 & 32 & $\mathbf{4 0}$ & 41 & 42 & $\mathbf{0 0}$ \\
02 & $\mathbf{2 0}$ & 01 & 12 & $\mathbf{3 0}$ & 11 & 22 & $\mathbf{4 0}$ & 21 & 32 & $\mathbf{0 0}$ & 31 & 42 & $\mathbf{1 0}$ & 41 \\
\hline $\mathbf{3 0}$ & 11 & 12 & $\mathbf{4 0}$ & 21 & 22 & $\mathbf{0 0}$ & 31 & 32 & $\mathbf{1 0}$ & 41 & 42 & $\mathbf{2 0}$ & 01 & 02 \\
11 & 12 & $\mathbf{4 0}$ & 21 & 22 & $\mathbf{0 0}$ & 31 & 32 & $\mathbf{1 0}$ & 41 & 42 & $\mathbf{2 0}$ & 01 & 02 & $\mathbf{3 0}$ \\
12 & $\mathbf{0 0}$ & 11 & 22 & $\mathbf{1 0}$ & 21 & 32 & $\mathbf{2 0}$ & 31 & 42 & $\mathbf{3 0}$ & 41 & 02 & $\mathbf{4 0}$ & 01 \\
\hline $\mathbf{1 0}$ & 21 & 22 & $\mathbf{2 0}$ & 31 & 32 & $\mathbf{3 0}$ & 41 & 42 & $\mathbf{4 0}$ & 01 & 02 & $\mathbf{0 0}$ & 11 & 12 \\
21 & 22 & 20 & 31 & 32 & 30 & 41 & 42 & 40 & 01 & 02 & 00 & 11 & 12 & 10 \\
22 & $\mathbf{3 0}$ & 21 & 32 & $\mathbf{4 0}$ & 31 & 42 & $\mathbf{0 0}$ & 41 & 02 & $\mathbf{1 0}$ & 01 & 12 & $\mathbf{2 0}$ & 11 \\
\hline $\mathbf{4 0}$ & 31 & 32 & $\mathbf{0 0}$ & 41 & 42 & $\mathbf{1 0}$ & 01 & 02 & $\mathbf{2 0}$ & 11 & 12 & $\mathbf{3 0}$ & 21 & 22 \\
31 & 32 & $\mathbf{0 0}$ & 41 & 42 & $\mathbf{1 0}$ & 01 & 02 & $\mathbf{2 0}$ & 11 & 12 & $\mathbf{3 0}$ & 21 & 22 & $\mathbf{4 0}$ \\
32 & $\mathbf{1 0}$ & 31 & 42 & $\mathbf{2 0}$ & 41 & 02 & $\mathbf{3 0}$ & 01 & 12 & $\mathbf{4 0}$ & 11 & 22 & $\mathbf{0 0}$ & 21 \\
\hline $\mathbf{2 0}$ & 41 & 42 & $\mathbf{3 0}$ & 01 & 02 & $\mathbf{4 0}$ & 11 & 12 & $\mathbf{0 0}$ & 21 & 22 & $\mathbf{1 0}$ & 31 & 32 \\
41 & 42 & $\mathbf{3 0}$ & 01 & 02 & $\mathbf{4 0}$ & 11 & 12 & $\mathbf{0 0}$ & 21 & 22 & $\mathbf{1 0}$ & 31 & 32 & $\mathbf{2 0}$ \\
42 & 40 & 41 & 02 & 00 & 01 & 12 & 10 & 11 & 22 & 20 & 21 & 32 & 30 & 31 \\
\hline
\end{tabular}

Figure 3: Latin square $\mathrm{T}$ obtained from $\mathrm{T}^{\prime}$ by applying the permutation $K_{b}^{-1} \circ L_{a}^{p}$ in each row $(a, b)$ of $\mathrm{T}^{\prime}$. In bold we emphasize those symbols where $\mathrm{T}^{\prime}$ and $\mathrm{T}$ differ.

Example 1 the permutation $\mathbf{T}_{(0,0),(0,2)}$ has five cycles of length three: $(x, 0) \rightarrow(x, 2) \rightarrow$ $(x, 1) \rightarrow(x, 0)$, for each $x \in\{0,1,2,3,4\}$.

\begin{tabular}{|l|l|l|l|l|l|l|l|l|l|l|l|l|l|l|}
\hline $\mathbf{0 0}$ & 01 & 02 & $\mathbf{1 0}$ & 11 & 12 & $\mathbf{2 0}$ & 21 & 22 & $\mathbf{3 0}$ & 31 & 32 & $\mathbf{4 0}$ & 41 & 42 \\
\hline 02 & $\mathbf{0 0}$ & 01 & 12 & $\mathbf{1 0}$ & 11 & 22 & $\mathbf{2 0}$ & 21 & 32 & $\mathbf{3 0}$ & 31 & 42 & $\mathbf{4 0}$ & 41 \\
\hline
\end{tabular}

However, the associated permutation in $\mathrm{T}$ is a cyclic permutation.

\begin{tabular}{|l|l|l|l|l|l|l|l|l|l|l|l|l|l|l|}
\hline $\mathbf{0 0}$ & 01 & 02 & $\mathbf{1 0}$ & 11 & 12 & $\mathbf{2 0}$ & 21 & 22 & $\mathbf{3 0}$ & 31 & 32 & $\mathbf{4 0}$ & 41 & 42 \\
\hline 02 & $\mathbf{2 0}$ & 01 & 12 & $\mathbf{3 0}$ & 11 & 22 & $\mathbf{4 0}$ & 21 & 32 & $\mathbf{0 0}$ & 31 & 42 & $\mathbf{1 0}$ & 41 \\
\hline
\end{tabular}

In the next result we prove that this situation occurs for each permutation $\mathrm{T}_{(a, b),\left(a^{\prime}, b^{\prime}\right)}$, whenever $K_{b, b^{\prime}}$ is a cyclic permutation.

Theorem 2 Let $n$ be an odd composite integer and let $p$ be the smallest prime divisor of $n$. Then, $\mathrm{T}$ defined above is a Latin square and

$$
\operatorname{pf}(\mathrm{T}) \geqslant \operatorname{pf}(K)\left(\frac{n}{p}\right)^{2} .
$$

Proof: We first prove that for each pair $\left(b, b^{\prime}\right)$ which is perfect in $K$ and for every $a$ and $a^{\prime}$ in $\Sigma_{L}$ (not necessarily distinct), the permutation $\mathrm{T}_{(a, b),\left(a^{\prime}, b^{\prime}\right)}$ is a cyclic permutation. 
It is not hard to see that the permutation $\mathrm{T}_{(a, b),\left(a^{\prime}, b^{\prime}\right)}$ is given by

$$
\mathrm{T}^{\prime}{ }_{(a, b),\left(a^{\prime}, b^{\prime}\right)}(x, y)=\left(L_{a, a^{\prime}}(x), P_{b, b^{\prime}}(y)\right) .
$$

Hence, symbols appearing in rows $(a, b)$ and $\left(a^{\prime}, b^{\prime}\right)$ of $\mathrm{T}^{\prime}$ can be ordered (by permuting columns if necessary) as

$$
\begin{array}{cl}
\mathrm{T}^{\prime}((a, b), \cdot):(x, y) & \left(L_{a, a^{\prime}}(x), P_{b, b^{\prime}}(y)\right) \cdots\left(L_{a, a^{\prime}}^{p-1}(x), P_{b, b^{\prime}}^{p-1}(y)\right) \cdots \\
\mathrm{T}^{\prime}\left(\left(a^{\prime}, b^{\prime}\right), \cdot\right):\left(L_{a, a^{\prime}}(x), P_{b, b^{\prime}}(y)\right) & \left(L_{a, a^{\prime}}^{2}(x), P_{b, b^{\prime}}^{2}(y)\right) \cdots\left(L_{a, a^{\prime}}^{p}(x), y\right) \cdots
\end{array}
$$

As $\mathrm{T}$ is obtained by modifying only symbols of the form $(x, \bar{P})$ we know that rows $(a, b)$ and $\left(a^{\prime}, b^{\prime}\right)$ in $\mathrm{T}$ are given by:

$$
\begin{array}{cl}
\mathrm{T}((a, b), \cdot):\left(\pi_{a, b}(x), \bar{P}\right) & \left(L_{a, a^{\prime}}(x), P_{b, b^{\prime}}(\bar{P})\right) \cdots\left(L_{a, a^{\prime}}^{p-1}(x), P_{b, b^{\prime}}^{p-1}(\bar{P})\right) \cdots \\
\mathrm{T}\left(\left(a^{\prime}, b^{\prime}\right), \cdot\right):\left(L_{a, a^{\prime}}(x), P_{b, b^{\prime}}(\bar{P})\right) & \left.\left(L_{a, a^{\prime}}^{2}(x), P_{b, b^{\prime}}^{2}(\bar{P})\right) \cdots\left(\pi_{a^{\prime}, b^{\prime}}\left(L_{a, a^{\prime}}^{p}(x)\right)\right), \bar{P}\right) \cdots
\end{array}
$$

From the definition of $L_{a}$ we get that

$$
L_{a^{\prime}}^{p}\left(L_{a, a^{\prime}}^{p}(x)\right)=\left(L_{a^{\prime}} \circ L_{a, a^{\prime}}\right)^{p}(x)=L_{a}^{p}(x) .
$$

Let $z=\pi_{a, b}(x)$. Then $K_{b}(z)=L_{a}^{p}(x)$ and we have that

$$
\pi_{a^{\prime}, b^{\prime}}\left(L_{a, a^{\prime}}^{p}(x)\right)=K_{b^{\prime}}^{-1} \circ L_{a^{\prime}}^{p}\left(L_{a, a^{\prime}}(x)\right)=K_{b^{\prime}}^{-1}\left(L_{a}^{p}(x)\right)=K_{b^{\prime}}^{-1} \circ K_{b}(z)=K_{b, b^{\prime}}(z) .
$$

So, after $p$ iterations of $\mathrm{T}_{(a, b),\left(a^{\prime}, b^{\prime}\right)}$ the symbol $(z, \bar{P})$ is transformed into $\left(K_{b, b^{\prime}}(z), \bar{P}\right)$. Hence, as $\left(b, b^{\prime}\right)$ is a perfect pair in $K$, the permutation $\mathrm{T}_{(a, b),\left(a^{\prime} b^{\prime}\right)}$ is cyclic.

It remains to show that $T$ is a Latin square. It is clear that the Cartesian product $\mathrm{T}^{\prime}$ is a Latin square. By the definition of $\mathrm{T}$ we only need to check that the modification $(x, \bar{P}) \rightarrow\left(\pi_{a, b}(x), \bar{P}\right)$ induces a permutation of $\Sigma_{\mathrm{T}}$ in each row and each column. By definition, the permutation $\pi_{a, b}$ is an injective function. Hence, the first components of the symbols in each row of $\mathrm{T}$ form a permutation of $\Sigma_{K}$. Therefore, each row of $\mathrm{T}$ is a permutation of $\Sigma_{\mathrm{T}}$. Let us assume that for some rows $(a, b),\left(a^{\prime}, b^{\prime}\right)$ and some column $(c, d)$ of $\mathrm{T}$ we have that

$$
\pi_{a, b}(L(a, c))=\pi_{a^{\prime}, b^{\prime}}\left(L\left(a^{\prime}, c\right)\right)
$$

and $P(b, d)=\bar{P}=P\left(b^{\prime}, d\right)$. Since $P$ is a Latin square we have that $b=b^{\prime}$ which implies that

$$
L_{a}^{p}(L(a, c))=L_{a^{\prime}}^{p}\left(L\left(a^{\prime}, c\right)\right) .
$$

From its definition $L_{a}^{-p} \circ L_{a^{\prime}}^{p}=L_{a^{\prime}, a}^{p}$ and $L\left(a^{\prime}, c\right)=L_{a, a^{\prime}}(L(a, c))$. Therefore,

$$
L(a, c)=L_{a^{\prime}, a}^{p} \circ L_{a, a^{\prime}}(L(a, c))=L_{a^{\prime}, a}^{p-1}(L(a, c)) .
$$

From this and the definition of $L$, the following equalities hold in $\mathbb{Z}_{n / p}$.

$$
L_{a, a^{\prime}}^{p-1}(L(a, c))=L(a, c)+(p-1)\left(a^{\prime}-a\right)=L(a, c) .
$$

Since $p$ is the smallest prime divisor of $n$, we know that $p-1$ does not divide $n / p$ which implies that $a=a^{\prime}$. Hence each column of $\mathrm{T}$ is a permutation of $\Sigma_{\mathrm{T}}$. Therefore $\mathrm{T}$ is a Latin square. This finishes the proof.

From the previous result we immediately get the following consequence. 
Corollary 1 For each odd composite integer $n$ we have that $\operatorname{pf}(n) \geqslant \operatorname{pf}(p, n / p)(n / p)^{2}$, where $p$ is the smallest prime divisor of $n$.

Proof: Let $K$ be a Latin rectangle of size $p \times(n / p)$ such that $\operatorname{pf}(K)=\operatorname{pf}(p, n / p)$ and let $\mathrm{T}$ be the Latin square of order $n$ defined above. Then, $\operatorname{pf}(n) \geqslant \operatorname{pf}(\mathrm{T}) \geqslant \operatorname{pf}(K)(n / p)^{2}=$ $\operatorname{pf}(p, n / p)(n / p)^{2}$.

\section{A graph theoretical Lemma}

When proving Theorem 3, we use induction on the size of Latin rectangles. The induction hypothesis will give us a Latin rectangle $K$ of size $p \times(n / p)$ such that $\operatorname{pf}(K) \geqslant p^{2} / 4$. By plugging this into Theorem 2 we obtain that $\operatorname{pf}(n, n) \geqslant n^{2} / 4$. In order to get a similar result for Latin rectangles, we have to choose some rows of the Latin square so that the resulting Latin rectangle has the desired number of perfect pairs. This last step can be achieved by using Lemma 1 which proves that a Latin square of order $n$ with more than $n^{2} / 4$ perfect pairs has $m$ rows such that the subrectangle induced by these rows has at least $m^{2} / 4$ perfect pairs. In fact, the property is slightly more general since it corresponds to a density property of a subgraph of a dense graph. For a graph $G=(V, E)$ we denote by $e(G)$ the cardinality of the set of edges $E$ and by $v(G)$ the cardinality of the set of vertices $V$. For each vertex $x$ of a graph $G$ we denote by $d_{G}(x)$ the degree of $x$ in $G$. Furthermore, we denote by $\delta(G)$ the minimum of $d_{G}(x)$ over all vertices $x$ in $G$.

Lemma 1 Let $G$ be a graph on $k$ vertices having $e(G)>k^{2} / 4$. Then, for $3 \leqslant m \leqslant k$, there is a subgraph $H$ of $G$ with $v(H)=m$ and $e(H)>m^{2} / 4$.

Proof: The case $3=m=k$ being obvious we can assume that $k \geqslant 4$. We proceed by induction on $k$. By deleting edges we can assume that $k^{2} / 4<e(G) \leqslant k^{2} / 4+1$.

We know that the minimum degree $\delta(G)$ of $G$ satisfies $\delta(G) k \leqslant 2 e(G)$. Hence, $\delta(G) \leqslant$ $k / 2+2 / k$. Let $v_{0}$ be a vertex of $G$ with $d_{G}\left(v_{0}\right)=\delta(G)$. Then, $e\left(G-v_{0}\right)=e(G)-\delta(G)$. For even $k, e(G)=k^{2} / 4+1$ so we have that

$$
e\left(G-v_{0}\right) \geqslant \frac{k^{2}}{4}+1-\frac{k}{2}-\frac{2}{k}=\frac{(k-1)^{2}}{4}+\frac{3}{4}-\frac{2}{k} .
$$

For $k \geqslant 4$ we have that $3 / 4-2 / k>0$ which implies $e\left(G-v_{0}\right)>(k-1)^{2} / 4$. For odd $k \geqslant 5$, we have that $e(G)>k^{2} / 4$ and also that $2 / k<1 / 2$, Hence, $\delta(G) \leqslant(k-1) / 2$ and the following inequalities hold.

$$
e\left(G-v_{0}\right)>\frac{k^{2}}{4}-\frac{k-1}{2}=\frac{(k-1)^{2}}{4}+\frac{1}{4}>\frac{(k-1)^{2}}{4} .
$$

Therefore, the subgraph $G-v_{0}$ has $k-1$ vertices and more than $(k-1)^{2} / 4$ edges. By the induction hypothesis, for each $m, 3 \leqslant m \leqslant k-1$ there is a subgraph $H$ of $G-v_{0}$ such that $v(H)=m$ and $e(H)>m^{2} / 4$. As $H$ is also a subgraph of $G$, we get the conclusion. 
The result is tight in the sense that we cannot replace the strict inequality in the hypothesis by equality even if we relax the conclusion in the same manner. In fact, the complete bipartite graph $K_{t, t}$ has $(2 t)^{2} / 4$ edges and $2 t$ vertices but any subgraph of $K_{t, t}$ with $2 t-1$ vertices has $t(t-1)$ edges which is less than $(2 t-1)^{2} / 4$.

\section{Induction argument}

Theorem 3 For each odd integer $n$ and each integer $m$, with $2 \leqslant m \leqslant n$, we have that $\operatorname{pf}(m, n) \geqslant m^{2} / 4$.

Proof: The case $m=2$ is direct since for each integer $n \geqslant 2$ the Latin rectangle with two rows, each of length $n$, where the second one is a cyclic rotation of the first one, has one perfect pair. For $3 \leqslant m \leqslant n$ we proceed by induction on $n$. The basis case, $n=3$, is obvious since there is a perfect Latin square of order 3 . We already know that when $n$ is an odd prime the result holds. So we assume that $n$ is odd and composite. Let $p$ be the smallest prime divisor of $n$. From Corollary 1 we know that $\operatorname{pf}(n, n) \geqslant \operatorname{pf}(p, n / p)(n / p)^{2}$. As $n / p$ is odd, less than $n$ and at least $p$, we can apply the induction hypothesis to get that $\operatorname{pf}(p, n / p) \geqslant p^{2} / 4$ which shows $\operatorname{pf}(n, n) \geqslant n^{2} / 4$.

Let $N$ be a Latin square of order $n$ such that $\operatorname{pf}(N) \geqslant n^{2} / 4$. Let $G$ be the graph on $\Sigma_{N}$ such that $a b$ is an edge of $G$ if and only if $N_{a, b}$ is a cyclic permutation. Then as $n$ is odd we get that $e(G)>n^{2} / 4$. From Lemma 1 we know that for each odd $m$, with $3 \leqslant m \leqslant n$, there is a subgraph $H$ with $e(H)>m^{2} / 4$ and $v(H)=m$. Therefore, the Latin rectangle $N^{\prime}$ obtained from $N$ by choosing rows whose labels are in the set of vertices of $H$ satisfies $\operatorname{pf}\left(N^{\prime}\right) \geqslant m^{2} / 4$. This shows that $\operatorname{pf}(m, n) \geqslant m^{2} / 4$.

\section{Conclusion}

In this work we have considered a quantitative version of Conjecture 1 focusing on uniform lower bounds for the function $\operatorname{pf}(n) / n^{2}$ : we have proved that $\operatorname{pf}(n) / n^{2} \geqslant \frac{1}{4}$, for each $n \geqslant 2$.

The proof of Theorem 3 suggests the following weakening of Conjecture 1.

Conjecture 2 For every two odd integers $n$ and $m$ such that $n \geqslant m \geqslant 3$ we have that $\operatorname{pf}(n) / n^{2} \geqslant \operatorname{pf}(m) / m^{2}$.

If true, then our lower bound $\frac{1}{4}$ for $\operatorname{pf}(n) / n^{2}$ might be replaced by $\frac{1}{3}$ when $n$ is odd. From the proof of Theorem 2 we can prove that $\operatorname{pf}(n, n) / n^{2} \geqslant \operatorname{pf}(p, n / p) / p^{2}$ when $p$ is a prime that divides $n$ and $n \geqslant p^{2}$. Hence, for $p$ a prime divisor of $n$ such that $p^{2} \leqslant n$ we have $\operatorname{pf}(n) / n^{2} \geqslant \operatorname{pf}(p) / p^{2}$ if the following is true.

Conjecture 3 For every odd $m$, we have that $\operatorname{pf}(m)=\operatorname{pf}(m, n)$, for each odd $n$ such that $n \geqslant m$. 


\section{References}

[1] B. A. Anderson, Finite topologies and Hamiltonian paths, J. Combin. Theory Ser. B 14, 87-93, 1973.

[2] D. Bryant, B. Maenhaut, I. M. Wanless, A family of perfect factorizations of complete bipartite graphs, J. Combin. Theory, Ser. A 98, 328-342, 2002.

[3] J. H. Dinitz, D. R. Stinson, Some new perfect one-factorizations from starters in finite fields, J. Graph Theory 13, 405-415, 1989.

[4] A. J. W. Hilton, M. Mays, C. A. Rodger, C. St. J. A. Nash-Williams, Hamiltonian double Latin squares, J. Combin. Theory Ser. B 87, 81-129, 2003.

[5] A. Kotzig, Hamilton graphs and Hamilton circuits, Theory of Graphs and its Applications (Proc. Sympos. Smolenice 1963) Nakl. CSAV, Praha 162, 1964.

[6] A. Kotzig and J. Labelle, Quelques problèmes ouverts concernant les graphes fortement hamiltoniens, Ann. Sc. Math. Québec 3, 95-106, 1979.

[7] E. Seah, Perfect one-factorizations of the complete graph-a survey, Bull. Inst. Combin. Appl. 1, 59-70, 1991.

[8] W. D. Wallis, One-factorizations, Math. Appl. 390, Kluwer, Dordrecht, 1997.

[9] D. G. Wagner, On the perfect one-factorization conjecture, Discrete Math. 104, 211215, 1992.

[10] I. M. Wanless, Perfect factorisations of bipartite graphs and Latin squares without proper subrectangles. Electron. J. Combin. 6, \#R9, 1999.

[11] I. M. Wanless, Cycle switches in Latin squares, Graphs Combin. 20, 545-570, 2004.

[12] I. M. Wanless and E. C. Ihrig, Symmetries that Latin squares inherit from 1factorizations, J. Combin. Designs 13, 157-172, 2005. 